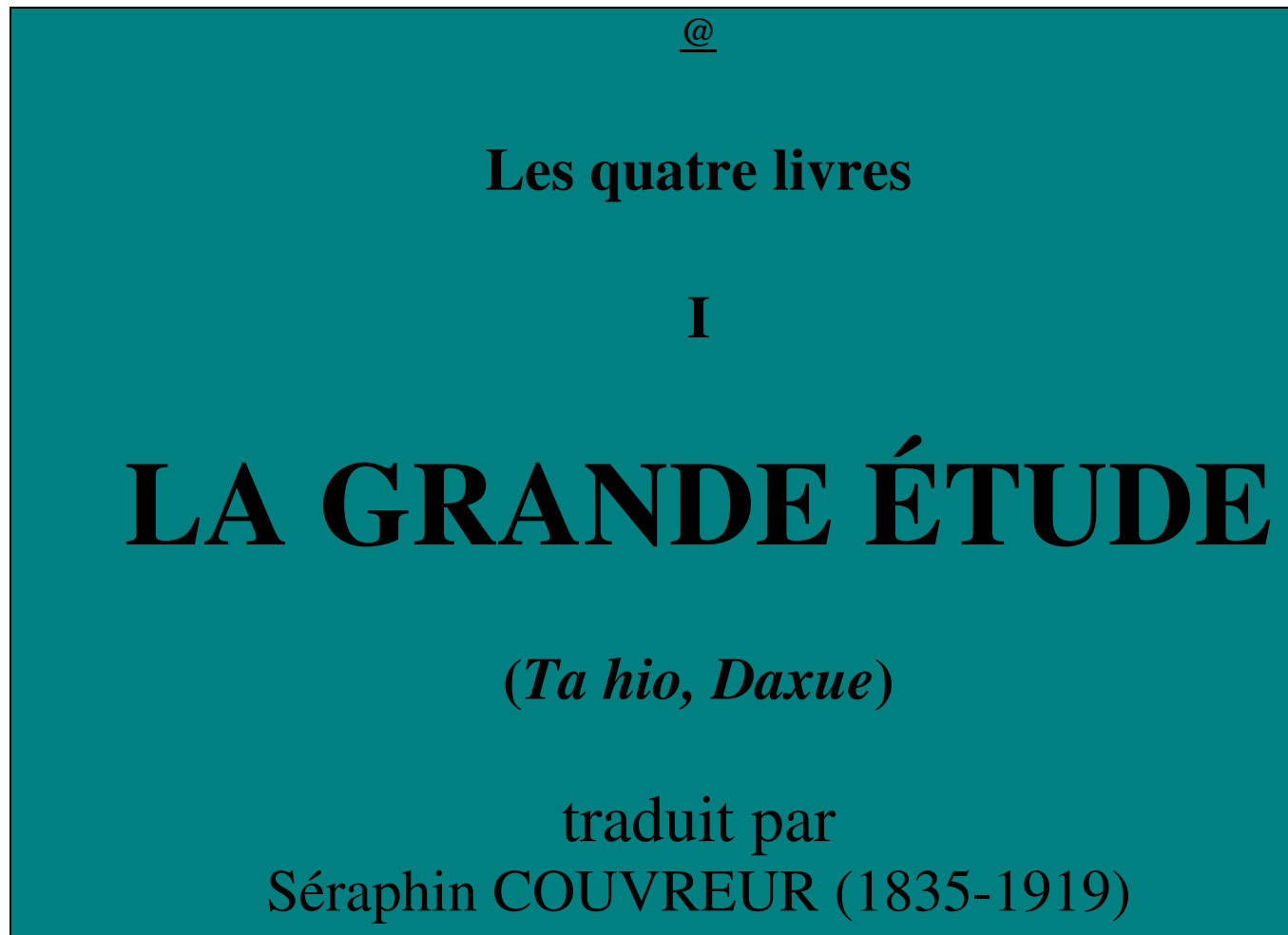

Un document produit en version numérique par Pierre Palpant, Collaborateur bénévole

Courriel : pierre.palpant@laposte.net

Dans le cadre de la collection : "Les classiques des sciences sociales" dirigée et fondée par Jean-Marie Tremblay, professeur de sociologie au Cégep de Chicoutimi

Site web : http ://www.uqac.uquebec.ca/zone30/Classiques_des_sciences_sociales/index.html

Une collection développée en collaboration avec la Bibliothèque Paul-Émile-Boulet de l'Université du Québec à Chicoutimi

Site web : http ://bibliotheque.uqac.uquebec.ca/index.htm 
Un document produit en version numérique par Pierre Palpant, collaborateur bénévole, Courriel : pierre.palpant@laposte.net

à partir de :

Les quatre livres

I. - La Grande Étude, traduit par Séraphin COUVREUR (1835-1919)

Club des Libraires de France, Paris, mai 1956, publié à partir de l'édition Les Humanités d'Extrême-Orient, Cathasia, série culturelle des Hautes Études de Tien-Tsin, LES BELLES LETTRES, Paris.

Polices de caractères utilisée : Times, 10 et 12 points, Mise en page sur papier format LETTRE (US letter), 8.5' $\mathrm{x} 11$ '.

Édition complétée le 30 novembre 2004 à Chicoutimi, Québec. 


\section{Table des matières}

$\underline{\text { Note CSS }}$

$\underline{\text { Paroles de Confucius }}$

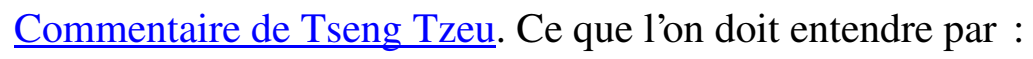

I. Faire briller les brillantes vertus

II. Renouveler les autres.

III. Se proposer pour terme la plus haute perfection

IV. La racine et les branches.

V. Scruter la nature des choses, perfectionner ses connaissances.

VI. Perfectionner sa volonté.

VII. Se perfectionner soi-même en réglant les mouvements de son cour.

VIII. Etablir l'ordre dans sa famille en se perfectionnant soi-même.

IX. Gouverner l'État en réglant sa famille.

$\mathrm{X}$. Un prince, en gouvernant bien sa principauté, procure la paix à tout l'empire. 


\section{NOTE C S S}

On se reportera utilement, pour la présentation de 'La Grande Etude', et plus généralement des 'Quatre Livres' et des livres classiques renfermant la doctrine de Confucius, aux éditions CSS suivantes :

- Confucius, par Edouard Chavannes.

- Histoire générale de la Chine, par Henri Cordier.

— La Chine antique, par Henri Maspero. Commentaires sur La Grande Etude, sur l'Invariable $\underline{\text { Milieu }}$

- La Pensée chinoise, par Marcel Granet. Commentaires sur Confucius, sur La Grande Etude, sur l'Invariable Milieu 


\section{A GR A N D E ÉT U D E}

Ce livre, tiré du Li ki, Mémorial des Usages et Cérémonies, se divise en deux parties. La première contient les paroles de Confucius transmises par son disciple Tseng tzeu, le philosophe Tseng. La seconde contient l'explication de Tseng tzeu écrite par ses disciples. Le texte a été revu, corrigé, et disposé dans l'ordre actuel par Tchou Hi.

\section{AVERTISSEMENT DE TCHOU HI}

Mon maître Tch'eng tzeu dit: "La Grande Etude est l'œuvre de Confucius et de ses disciples. Elle est comme la porte qui ouvre la voie de la vertu. L'ordre anciennement suivi dans les études n'est plus connu à présent que par ce livre, qui heureusement nous a été conservé, et par le Liun iu et les écrits de Meng tzeu, qui sont venus ensuite. Certainement, le disciple de la sagesse qui commencera par l'étude de ce livre ne sera pas exposé à s'égarer. » 


\section{PAROLES DE CONFUCIUS}

La voie de la Grande Étude consiste en trois choses, qui sont de faire briller en soi-même les vertus brillantes que la nature met dans l'âme de chacun, de renouveler les autres hommes, et de se fixer pour terme la plus haute perfection.

Connaissant le terme où l'on doit tendre et s'arrête $r$, on peut prendre une détermination. Cette détermination étant prise, l'esprit peut avoir le repos. L'esprit, étant en repos, peut jouir de la tranquillité. Jouissant de la tranquillité, il peut examiner les choses. Après cet examen, on peut atteindre le but, qui est la perfection.

En toute chose il faut distinguer le principal et l'ac cessoire et, dans les affaires, la fin et le commencement. Celui qui sait mettre chaque chose en son rang n'est pas loin de la voie de la Grande Étude ou de la perfection.

Les anciens princes, pour faire briller les vertus naturelles dans le cœur de tous les hommes, s'appliquaient auparavant à bien gouverner chacun sa principauté. Pour bien gouverner leurs principautés, ils mettaient auparavant le bon ordre dans leurs familles. Pour mettre le bon ordre dans leurs familles, ils travaillaient auparavant à se perfectionner eux-mêmes, ils réglaient auparavant les mouvements de leur cœur. Pour régler les mouvements de leur cœur, ils rendaient auparavant leur volonté parfaite. Pour rendre leur volonté parfaite, ils développaient leurs connaissances le plus possible. On développe ses connaissances en scrutant la nature des choses.

La nature des choses une fois scrutée, les connaissances atteignent leur plus haut degré. Les connaissances étant arrivées à leur plus haut degré, la volonté devient parfaite. La volonté étant parfaite, les mouvements du cœur sont réglés. Les mouvements du cœur étant réglés, tout homme est exempt de défauts. Après s'être corrigé soi-même, on établit l'ordre dans la famille. L'ordre régnant dans la famille, la principauté est bien gouvernée. La principauté étant bien gouvernée, bientôt tout l'empire jouit de la paix.

Depuis le Fils du Ciel jusqu'au plus humble particulier, chacun doit avant tout se perfectionner soi-même. Celui qui néglige le principal ne peut régler convenablement les choses qui en dépendent. Jamais un homme qui soigne peu ce qu'il doit aimer le plus n'a gouverné avec diligence ce qui lui est moins cher. 
La Grande Etude est l'étude de ceux qui ne sont plus enfants. Les vertus brillantes sont celles que l'homme reçoit du Ciel en naissant. Il les reçoit libres d'entraves, lumineuses, exemptes de ténèbres, afin que par elles il connaisse tous les principes, et règle sa conduite en toutes choses. Mais, dans les liens d'un corps composé d'éléments matériels, au milieu des ténèbres amassées par les passions humaines, parfois elles s'obscurcissent. Néanmoins, la lumière qui est inhérente â leur nature ne s'éteint jamais entièrement. C'est pourqu oi le disciple de la sagesse doit se servir de la lumière qu'elles donnent encore, afin de les faire briller, et de leur rendre leur premier éclat. Le sage, après avoir fait briller en lui-même ses brillantes vertus, doit étendre son action aux autres hommes, et faire en sorte qu'ils se débarrassent des impuretés qui les souillent depuis longtemps. Les brillantes vertus sont la bienveillance, la justice, le sentiment des convenances, la prudence et la sincérité, que le Ciel met dans le cour de chaque homme, et qu'on appelle les cinq règles principales. 


\section{COMMENTAIRES DE TSENG TZEU}

Le chapitre précédent contient les paroles de Confucius rapportées par Tseng tzeu. Les dix chapitres d'explication se composent des idées de Tseng tzeu transmises par ses disciples. Dans les anciens exemplaires, beaucoup de tablettes n'étaient pas à leur place. M'ap puyant sur les décisions de Tch'eng tzeu, j’ai révisé le texte du livre, et disposé les parties du commentaire dans l'ordre suivant.

Le commentaire contient en tout dix chapitres. Dans les quatre premiers, l'auteur explique le sens et le but des principes généraux et, dans les six derniers, le travail que demandent les règles particulières. Dans le cinquième, il fait connaître la nécessité de discerner le bien et, dans le sixième, le fondement de la perfection. Ces deux chapitrer exigent des commençants une attention spéciale. Le lecteur ne doit pas les mépriser à cause de leur simplicité. 


\section{CHAPITRE I}

Ce premier chapitre du commentaire explique ce qu'on doit en tendre par "faire briller les brillantes vertus».

$\underline{\text { Li ki, XXXIX, } 9}$

Dans le Chou king, au chapitre intitulé « K'ang kao ${ }^{1} »$, il est dit :

W enn wang fut capable de faire briller ses vertus naturelles.

Dans le Chou king, au chapitre intitulé « T'ai kia ${ }^{2} »$, il est dit :

Tch'eng T'ang, votre prédécesseur, veillait sur ces dons britlants du Ciel.

Dans le Chou king, au chapitre intitulé «Institutions de l'Empereur Iao », il est dit :

Il fut capable de faire briller ses vertus ém inentes.

Tous ces princes faisaient briller leurs vertus.

1 Avis donnés à K'ang chou par son frère Ou ouang.

2 Avis donnés à l'empereur T'ai kia par I in, son ministre. 


\section{CHAPITRE II}

Ce deuxième chapitre du commentaire explique ce qu'on doit entendre par

«renouveler les autres ».

$\underline{\text { Li ki, XXXIX, } 10}$

La baignoire de l'empereur Tch'eng t'ang portait cette inscription :

Renouvelez-vous enfin véritablement, renouvelez-vous chaque

jour, et ne cessez de vous renouveler.

Les souillures du coeur se lavent comme celles du corps: Quand un homme est enfin parvenu à laver les souillures invétérées du coeur, et à se renouveler courageusement, il doit continuer chaque jour à se renouveler, à l'aide de ce qu'il a déjà renouvelé en lui.

Dans les Avis donnés à K’ang chou il est dit :

Encouragez le peuple à se renouveler.

Dans le Cheu king il est dit :

Bien que la principauté de T cheou soit ancienne, ses princes ont

reçu du Ciel un $m$ andat nouveau pour com $m$ ander à tout l'em pire.

Dans la troisième partie du Cheu king, qui est intitulée Ta ia, au chapitre concernant Wenn wang, il est dit que Wenn wang, prince de Tcheou, s'étant renouvelé lui-même par la pratique de la vertu, et ayant déterminé le peuple à suivre son exemple, sa famille, qui était en possession d'une principauté ancienne, reçut du Ciel un mandat nouveau.

Pour cette raison, les princes donnent à chaque chose toute leur application. 


\section{CHAPITRE III}

Ce troisième chapitre du commentaire explique ces mots

" se proposer pour terme la plus haute perfection».

Li ki, XXXIX, 11>12

\section{Le Cheu king dit :}

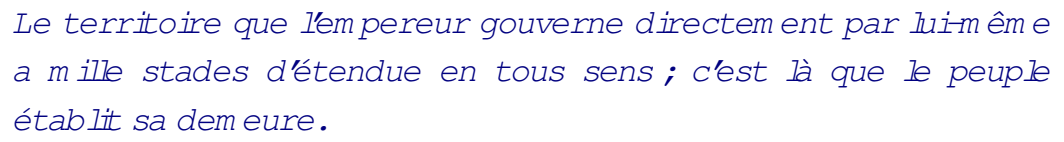

Le Cheu king dit :

L'oiseau jaune ${ }^{1}$ qui crie m ièn mân se tient à l'angle dune colline.

Le Philosophe dit :

L'oiseau jaune sait le lieu où il doit se fixer. Se peut-il quinn hom me soit moins intelligent quin oiseau ?

Chaque chose a un lieu déterminé où elle doit se fixer. Le territoire soumis à la juridiction directe de l'empereur a mille stades d'étendue en tous sens. C'est le lieu où tous les sujets de l'empire aiment à fixer leur demeure. L'oiseau jaune, qui est un être dépourvu de raison, sait néanmoins le lieu où il doit s'arrêter. Si l'homme, qui seul entre tous les êtres est doué d'intelligence, ne sait pas choisir et prendre pour terme la plus haute perfection, il est pire qu'un oiseau.

\section{Dans le Cheu king il est dit :}

$Q$ ue la vertu de $W$ enn wang fut sublime ! Il brilla constamm ent par le soin qu'ileut de tendre au plus parfait.

Il eut toujours pour terme de ses actions, comme prince de Tcheou, la bienfaisance, comme vassal des In, la soumission, comme fils, la piété filiale, comme père, la bonté, comme concitoyen, la bonne foi.

\section{Il est dit dans le Cheu king :}

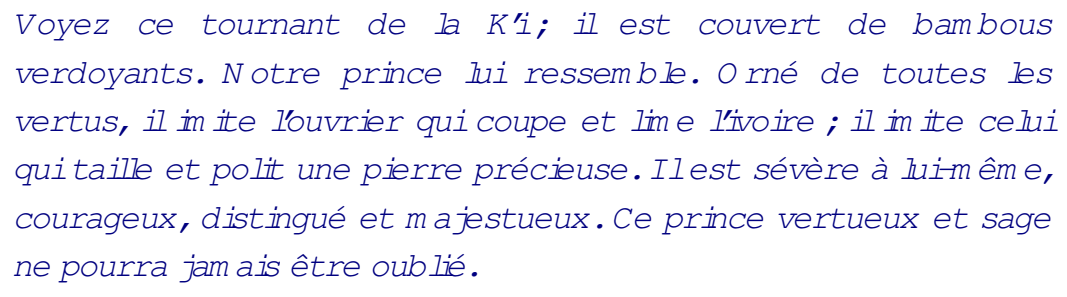

\footnotetext{
${ }^{1}$ Le loriot.
} 
«Imiter l'ouvrier qui coupe et lime l'ivoire », c'est s'appliquer à l'étude de la sagesse. «Imiter celui qui taille et polit les pierres précieuses », c'est se perfectionner soi-même. «Il est sévère à lui-même et courageux », c'est-à-dire très attentif à bien faire ; "distingué et majestueux », c'est-à-dire inspirant le respect et digne d'être imité. «Ce prince sage et vertueux ne pourra jamais être oublié », ces paroles signifient que sa vertu est parfaite, et restera toujours dans la mémoire du peuple.

Il est dit dans le Cheu king :

Les anciens rois ${ }^{1}$ ne seront pas oubliés.

Les princes venus après eux ont profité de leur sagesse et ont transmis comme eux le pouvoir impérial à leurs descendants. Le peuple a joui de la paix et des autres avantages que ces rois lui ont procurés. Aussi leur mémoire leur a-t-elle survécu.

${ }^{1}$ Wenn wang et Ou wang. 


\section{CHAPITRE IV}

Ce quatrième chapitre du commentaire explique ce qu'on doit entendre par

«la racine et les branches ».

$\underline{\text { Li ki, XXXIX, } 13}$

Confucius disait :

- Juger les procès, je le pourrais tout comme un autre. Mais assurément, faire qu'il n'y eût plus de procès, ne serait -ce pas le mieux ? Débouter de leurs prétentions les plaideurs peu sincères, inspirer au peuple une grande horreur des chicanes, c'est connaître le principal devoir de l'homme, le devoir de se perfectionner lui même, pour réformer les autres. 


\title{
CHAPITRE V
}

Li ki, XXXIX, 14

\author{
C'est connaître le principal devoir de l'homme ; c'est la plus haute \\ science.
}

Le chapitre cinquième du commentaire de Tseng tzeu était l'explication de ces deux expressions "scruter la nature des choses, perfectionner ses connaissances »; à présent il n'existe plus. Dernièrement, pour y suppléer, moi Tchou Hi, je me suis permis de prendre et d'ajouter l'explication de Tch'ong tzeu ; la voici. En disant que l'homme, pour perfectionner ses connaissances, doit scruter la nature des choses, Confucius enseigne que, si nous voulons étendre nos connaissances le plus possible, il faut examiner les choses et chercher leur raison d'être. Il n'est personne dont l'intelligence ne puisse acquérir des connaissances, et il n'est rien sur la terre qui n'ait sa raison d'être. Mais celui qui n'a pas entièrement approfondi la raison des choses ne la connaît qu’imparfaitement. Aussi, la Grande Etude, dès le début, avertit l'étudiant d'examiner toutes les choses avec lesquelles il est en contact, de se servir de la connaissance qu'il en a déjà pour pénétrer davantage leur raison d'être, de con tinuer ses recherches jusqu'aux dernières limites. Quand il aura longtemps fait tout ce qui est en son pouvoir, et qu'un beau matin il aura tout compris parfaitement, l'extérieur et l' intérieur des choses, les points les plus subtils comme les plus apparents, tout lui sera connu. Les principes innés dans l'âme et leurs applications n'auront plus pour lui d'obscurité. Cela .t'appelle "avoir pénétré la nature des choses»; cela s'appelle «le plus haut point de la connaissance ». 


\section{CHAPITRE VI}

Ce sixième chapitre du commentaire explique ce qu'on doit entendre par ces mots "perfectionner sa volonté ».

Ce que Confucius appelle «rendre sa volonté parfaite », c'est ne pas se tromper soi-même; comme avoir en aversion une odeur fétide, aimer une chose vraiment belle, c'est ne pas se tromper. Cela s'appelle trouver sa parfaite satisfaction en soi-même. Aussi le sage veille-t-il attentivement sur ce que lui seul connaît ${ }^{1}$.

Le disciple de la sagesse, après avoir scruté la nature des choses et perfectionné ses connaissances, a les lumières nécessaires pour faire le bien et éviter le mal. S'il ne sait pas déployer une véritable énergie, il se trompe lui-même.

Lorsqu'un homme vicieux se trouv e seul, il commet le mal ; il n'est rien qu'il ne se permette. S’il aperçoit un homme sage, aussitôt. il dissimule, cache sa méchanceté, et se montre vertueux.

Mais l'homme sage pénètre ses intentions, comme s’il voyait le fond de son cour. Que sert alors cette dissimulation? C'est ce que dit le proverbe :

L'intérieur se manifeste toujours à l'extérieur.

Aussi le sage a-t-il grand soin de veiller sur ses pensées et ses actions les plus secrètes.

Tseng tzeu dit :

- Ce que tous les yeux voient, ce que tout le monde montre du doigt, n'exige-t-il pas toute notre attention?

La richesse d'une famille se voit aux ornements de la maison. De même, la vertu d'un homme paraît dans toute sa personne ; la dilatation de son cour rejaillit sur son corps. C'est pourquo i le disciple de la sagesse a soin de rendre sa volonté parfaite.

\footnotetext{
${ }^{1}$ Ses pensées et ses actions les plus secrètes.
} 


\section{CHAPITRE VII}

Ce septième chapitre du commentaire explique ce qu'on doit entendre par ces mots
« se perfectionner soi-même en réglant les mouvements de son caur ».

$\underline{\text { Li ki, XXXIX, } 15}$

Ces paroles, «l'homme se perfectionne en réglant les mouvements de son coaur », signifient que le coar n'est pas réglé, mais agité et troublé par la passion, lorsqu'il est sous l'impression de la colère ou du ressentiment, de la crainte ou de la, terreur, ni lorsqu'il est dans les liens d'une affection ou d'un violent attachement, dans l'inquiétude ou l'affliction. Quand le cour s'en va où sa passion l'entraîne, on écoute et on n'entend pas, on mange et on ne perçoit pas le goût de la nourriture. Tel est le sens de ces paroles, «l'homme se perfectionne en réglant les mouvements de son cour $»$. 


\section{CHAPITRE VIII}

Ce huitième chapitre du commentaire explique ces mots "établir l'ordre dans sa famille en se perfectionnant soi-même ».

Li ki, XXXIX, 16

Ces paroles, «établir le bon ordre dans sa famille en se perfectionnant soimême », signifient que l'homme est injuste et partial envers les objets de sa tendresse ou de son affection, de son mépris ou de son aversion, de sa vénération ou de son respect, de sa commisération ou de sa pitié, de son dédain ou de son dégoût. Aussi peu d'hommes connaissent les défauts de ceux qu'ils aiment, ou les bonnes qualités de ceux qu'ils ont en aversion. Le proverbe dit :

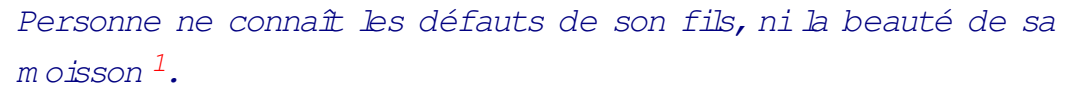

Tel est le sens de ces paroles : « nul ne peut mettre l'ordre dans sa famille, s’il ne s'applique à se perfectionner lui -même. »

\footnotetext{
${ }^{1}$ Le laboureur trouve toujours que sa moisson n'est pas belle.
} 


\section{CHAPITRE IX}

Ce neuvième chapitre du commentaire explique cette sentence "gouverner l'État en réglant sa famille».

$\underline{\text { Li ki, XXXIX, 17>21 }}$

«Pour bien gouverner un Etat, il faut d'abord établir le bon ordre dans sa propre famille », ces paroles de Confucius signifient qu'un prince incapable d'instruire les personnes de sa maison est incapable d'instruire les autres. Le sage, sans sortir de sa famille, répand l’ins truction dans la contrée par son exemple. Car le citoyen doit obéir à son prince comme le fils à son père, et aux officiers, comme le frère puîné obéit à son frère aîné ; le prince doit commander à ses sujets avec la même bonté qu'un père à ses enfants.

Ou wang donne cet avis à K’ang chou :

- Ayez la sollicitude d'une mère pour son fils nouveau -né.

Une mère cherche sérieusement à deviner les désirs de son fils ; elle devine juste, ou peu s'en faut. jamais femme, avant de se marier, n'eut besoin d'apprendre à élever des enfants. Une seule famille dont les membres s'entr'aident avec affection porte par son exemple toute la nation à exercer la bienfaisance. Une seule famille dont les membres sont polis et condescendants entre eux fait fleurir la. politesse et la condescendance parmi tous les concitoyens. La vie licencieuse et la perversité d'un seul homme mettent l'insurrection et le désordre dans tout le peuple. Tant est grande l'influence de la vertu ou du vice! L'adage dit :

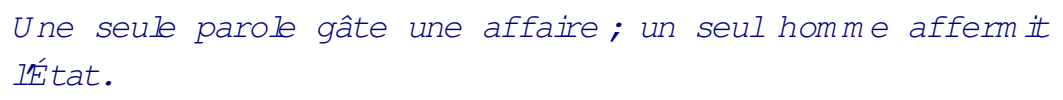

Iao et Chouenn ont conduit l'empire par la voie de la bie nfaisance, et le peuple les a suivis. Kie et Tcheou ont conduit l'empire par la voie de la violence, et le peuple les a suivis. Si les ordres du prince sont en contradiction avec sa conduite, le peuple n'obéit pas. Un prince sage, avant d'exiger une chose des autres, la pratique d'abord lui -même ; avant de reprendre un défaut dans les autres, il a soin de l'éviter lui-même. Un homme qui ne sait pas mesurer et traiter les autres avec la. même mesure que lui-même ne peut pas les instruire. C'est donc en rég lant sa maison qu'un prince arrive à bien gouverner.

Il est dit dans le Cheu king :

Le pêcher est délicat et beau; son feuillage est verdoyant. Ces

jeunes filles, profitant de la saison, vont célébrer leurs noces

chez leurs fiancés. Elles agiront convenablement envers les

personnes de leurs nouvelles fam illes. 
Le sage traite convenablement les personnes de sa maison ; il peut ensuite instruire ses concitoyens. On lit dans le Cheu king :

Vous agissez convenablem ent envers vos frères, soit plus âgés, soit m oins âgés que vous.

Le sage agit convenablement envers tous ses frères; il peut ensuite instruire ses concitoyens. Le Cheu king dit :

Sa conduite envers tous est irréprochable; il régira tous les peuples de l'empire.

Le sage remplit d'une manière exempla ire ses devoirs de père, de fils, de frère plus âgé et de frère moins âgé ; et le peuple l'imite. Voilà le sens de ces paroles : «Un prince, pour bien gouverner ses Etats, doit établir le bon ordre dans sa maison. » 


\title{
CHAPITRE X
}

\author{
Ce dixième chapitre du commentaire explique comment \\ « un prince, en gouvernant bien sa principauté, procure la paix à tout l'empire».
}

Li ki, XXXIX, 22>41

Voici le sens de ces paroles : «Un prince fait régner la paix dans tout l'empire en gouvernant bien sa principauté. » Si le prince honore ses parents, le peuple pratiquera la piété filiale. Si le prince respecte ses aînés, le peuple pratiquera le respect envers les aînés. Si le prince a compassion des orphelins, le peuple fera de même. Ainsi un prince sage a une règle pour juger. Ne faites pas à vos inférieurs ce qui vous déplaît de la part de vos supérieurs, ni à vos supérieurs ce qui vous déplaît de la part de vos inférieurs. Ne faites pas à ceux qui vous suivent ce qui vous déplaît de la part de ceux qui vous précèdent, ni à ceux qui vous précèdent ce qui vous déplaît de la part de ceux qui vous suivent. Ne faites pas à ceux qui sont à votre gauche ce qui vous déplaît de la part de ceux qui sont à votre droite, ni à ceux qui sont à votre droite ce qui vous déplaît de la part de ceux qui sont à votre gauche. C'est ce qui s'appelle une règle pour juger.

Dans le Cheu king il est dit :

Notre aim able prince est le père du peuple.

Etre le père du peuple, c'est aimer ce qui plaît au peuple, et a voir en aversion ce qui lui déplaît. Il est dit dans le Cheu king :

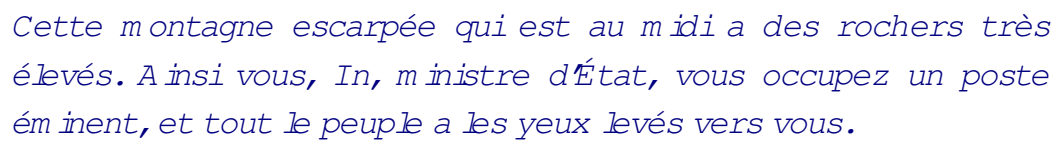

Celui qui tient les rênes du gouvernement doit, en raison de sa dignité, être sur ses gardes. Sil com met une faute, chacun lui prodigue l'outrage.

Il est dit dans le Cheu king :

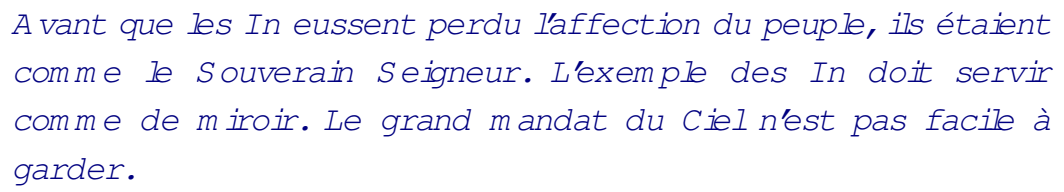

Cela veut dire qu'on obtient l'empire en obtenant l'affection du peuple, et qu'on perd l'empire en perdant l'affection du peuple.

Pour cette raison, le sage s'applique avant tout à pra tiquer la vertu. Celui qui a la vertu a l'affection des hommes ; celui qui a l'affection des hommes possède la terre ; celui qui possède la terre a des richesses ; celui qui a des richesses a les ressources nécessaires. La vertu est comme la racine; les richesses sont comme les branches. Exclure de ses pensées la vertu, et ne 
travailler qu'à s'enrichir, c'est disputer au peuple ses biens et autoriser la rapine par son exemple. Si le prince amasse des richesses, le peuple se disperse. Si le prince laisse les richesses partagées entre ses sujets, le peuple se groupe autour de lui. Une parole contraire à la justice rencontre dans le peuple une résistance injuste. Les richesses acquises par des moyens injustes s'écoulent par de s voies injustes.

Dans le Chou king, Ou wang dit à son frère K’ang chou :

- L'empereur ne reçoit pas le mandat du Ciel pour toujours.

Ces paroles signifient que, si le prince est vertueux, il obtient le mandat du Ciel; s'il devient mauvais, il le perd. Il est dit dans les annales de la principauté de Tch'ou :

La nation de Tch'ou n'attache pas un grand prix à l'or ni aux pierreries; elle n'estim e que la probité.

Fan, oncle maternel de Wenn, prince de Tsin, dit :

- L'exilé ${ }^{1}$ estime la piété filiale, et non les richesses et les honneurs.

Dans le Chou king, le prince de Ts’in dit à ses soldats :

$S$ 'il y avait un ministre d'État qui eût pour toutes qualités la simplicité, la probité, et dont le cou ur fût exempt de passions; qui pût en quelque sorte faire siennes les qualités de tous les autres; qui, voyant des hommes de talent, se réjouit comme s'il avait hii-même leurs talents; qui, voyant des hom mes savants et vertueux, les aimât sincèrement; qui ne se contentât pas de louer de bouche, $m$ ais considérât vraiment comme siennes les qualités des autres, et pût protéger mes descendants et tout le peuple, un tel homme serait très utile. Au contraire, si un $m$ inistre d'́tat, voyant des hommes de talent, leur porte envie et les a en aversion; si, voyant des hommes savants et vertueux, il leur fait de l'opposition et empêche qu'on ne les connaisse; s'il ne peut faire siennes les qualités des autres, ni par conséquent protéger mes descendants et $m$ on peuple, il est m êm e dangereux à létat.

Un prince vertueux l'éloignerait, l'enverrait en exil, le reléguerait au milieu des étrangers qui entourent le pays. Il ne lui permettrait pas de partager avec les autres citoyens le séjour de la Chine. C'est ce qu'on expri me en disant que seul l'homme vertueux sait aimer et haïr comm e il faut.

Connaître un homme probe et capable, et ne pas vouloir l'élever aux charges, ou le promouvoir tard, c'est négligence. Connaître un homme vicieux

\footnotetext{
${ }^{1}$ C'est-à-dire Wenn, prince de Tsin, qui était alors en exil.
} 
et ne pas vouloir le chasser, ou le chasser à peu de distance, c'est une indulgence excessive.

Aimer ce que les autres n'aiment pas, ne pas aimer ce qu'ils aiment, c'est être en opposition avec la nature humaine. C'est attirer infailliblement des malheurs sur sa personne. Il existe pour les princes une excellente règle de conduite qui est d'aimer ce qu'a ime le peuple, et d'avoir en aversion ce quil n'aime pas. Ils la gardent, quand ils sont bons et sincères ; ils la violent, quand ils sont orgueilleux et amis du faste. Pour procurer des ressources à l'État, il est un excellent moyen. Quand ceux qui les procurent sont nombreux, et ceux qui les consomment en petit nombre, quand ceux qui les obtiennent par leur travail agissent avec promptitude, et ceux qui les emploient avec lenteur, elles sont toujours plus que suffisantes. .

Un prince bienfaisant augmente sa puissance par sa libéralité ; celui qui n'est pas bienfaisant augmente ses richesses au détriment de son crédit et de son autorité. Quand le prince aime à faire du bien, toujours ses sujets aiment à remplir leurs devoirs envers lui. Quand les sujets aiment à remplir leurs devoirs envers le prince, les affaires du prince sont toujours menées à bonne fin. Les richesses amassées dans les magasins et les trésors publics restent toujours au prince, parce qu'elles ne sont pillées par personne.

Meng Hien tzeu ${ }^{1}$ dit :

- Celui qui entretient des attelages de quatre chevaux ne doit pas s'occuper de poules et de pourceaux, comme le font les hommes du bas peuple. Une famille ${ }^{2}$ qui emploie la glace pour conserver les viandes ${ }^{3}$ ne doit pas nourrir des boufs et des b rebis, comme font les bergers et les laboureurs. Une famille de ministre d'État, qui entretient pour la guerre cent attelages de quatre chevaux, ne doit pas nourrir des ministres qui lèvent des tributs exorbitants. Il vaudrait mieux qu'elle eût des ministres voleurs que des exacteurs.

C'est ce que l'on exprime en disant :

La justice est beaucoup phus profitable à létat que les revenus.

Si celui qui administre les affaires publiques s'appli que principalement à amasser des trésors, la faute en est à des ministres indignes. Il les croit hommes de bien. Quand des ministres méprisables ont le maniement des affaires publiques, il en résulte de grands malheurs et de grands dommages. Quand même il resterait des hommes vertueux, il leur serait impossible de remédier au mal. C'est ce qu'on exprime en disant : «La justice est beaucoup plus profitable à l'État que les revenus. »

$$
* * *
$$

$\underline{\text { Paroles de Confucius }}$

\footnotetext{
${ }^{1}$ Sage préfet de la principauté de Lou.

2 De ministre d'Etat, de grand préfet ou de prince.

${ }^{3}$ Lorsqu'elle célèbre des funérailles ou fait des offrandes.
} 


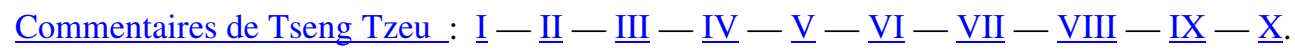


Nom du document : la_grande_etude.doc

Dossier :

C:\CSS\Envoi021204\classiques

Modèle :

$\mathrm{C}:$ IWINDOWS \Application

DatalMicrosoft $\backslash$ Modèles $\backslash$ Normal.dot

Titre :

La Grande Etude

Sujet :

série Chine

Auteur :

divers

Mots clés :

Daxue, Ta hio, Ta Hsüeh, Great Learning, Si Shu, Four

Books, Confucian Classics, confucianisme, confucius, confucianisme, confucius, civilisation chinoise, pensee chinoise, Chine ancienne, Chine antique, Chine classique, philosophie chinoise

Commentaires : iences_sociales/index.html

http://www.uqac.uquebec.ca/zone30/Classiques_des_sc

Date de création : $\quad$ 18/11/04 13:38

$\mathrm{N}^{\circ}$ de révision : 6

Dernier enregistr. le : 01/12/04 20:31

Dernier enregistrement par : Pierre Palpant

Temps total d' édition5 Minutes

Dernière impression sur : $\quad 05 / 12 / 04$ 11:12

Tel qu' à la dernière impression

Nombre de pages :

23

Nombre de mots : 4271 (approx.)

Nombre de caractères : 24349 (approx.) 